\title{
THE ASSOCIATION BETWEEN AUTOANTIBODY LEVELS AND THE OUTCOMES OF ANTI-TUMOUR NECROSIS FACTOR ALPHA TREATMENT IN RHEUMATOID ARTHRITIS - A RETROSPECTIVE COHORT STUDY WITH TWO YEARS FOLLOW-UP
}

Daniela Oliveira1, Ana Martins ${ }^{1, \star}$, Frederico Martins², Maria Rato ${ }^{1}$, Filipe Pinheiro ${ }^{1}$, Diogo Fonseca ${ }^{3}$, Salomé Garcia ${ }^{1}$, Bruno Fernandes ${ }^{1}$, Carlos Vaz ${ }^{1}$, Miguel Bernardes ${ }^{1}$, Lúcia Costa ${ }^{1}$

1.Centro Hospitalar Universitário de São João, Porto (Douro Litoral), Portugal; 2.Centro Hospitalar Universitário do Algarve, Faro (Algarve), Portugal; 3.Centro Hospitalar de Vila Nova de Gaia/Espinho, Vila Nova de Gaia (Douro Litoral), Portugal.

*Corresponding author: danielasoff@gmail.com

\section{BACKGROUND}

In rheumatoid arthritis (RA), autoantibodies namely anticitrullinated protein antibodies (anti-CCP) have prognostic value, independently predicting radiologic progression. However, the evidence is still controversial about how the autoantibody levels change over time and their role in treatments outcomes and in monitoring disease activity in RA. This study aimed to characterize the changes of autoantibodies levels (rheumatoid factor [RF] and anti-CCP) over time and to explore the association between these autoantibodies and the outcomes of the first anti-tumor necrosis factor alfa (anti-TNF- $\alpha$ ) therapy as first biologic agent in RA.

\section{METHODS}

An observational retrospective cohort study was conducted with two years of follow-up. Patients with diagnosis of RA according to American College of Rheumatology (ACR) criteria and registered on Rheumatic Diseases Portuguese Register (Reuma.pt) who started their first anti-TNF- $\alpha$ agent (as first biologic) between 2003 and 2018 were included. Patients with positive RA (> $30 \mathrm{UI} / \mathrm{mL}$ ) and/or positive anti-CCP (>10 U/mL) at their first visit were included. Demographic, clinical and laboratory data were obtained by consulting Reuma.pt. Disease activity score for 28 joints (DAS28 [3v]; DAS28 [4v]; DAS28 [3v; C-reactive protein \{CRP\}], DAS28 [4v; $\mathrm{CRP}$ ], delta DAS28 [4v]), health assessment questionnaire (HAQ), delta HAQ, anti-CCP and RF levels were assessed at baseline, 12 and 24 months. To examine the differences between anti-CCP and RF levels at baseline, 12 months and 24 months the Wilcoxon test for paired samples was performed. In order to correlate the anti-CCP and RF levels with DAS28 variables, delta DAS28 (4v), HAQ and delta HAQ at baseline, 12 months and 24 months, a correlation coefficient, Spearman's coefficient, was used.

\section{RESULTS}

A total of 116 patients (mean age of $50.2 \pm 10.4$ years old; $85.3 \%$ female) with RA were included with a median disease duration of 10.5 (5-18.5) years and a follow-up time of 8 (5-14) years. About $49 \%$ of patients were FR and anti-CCP positivity, $38 \%$ only FR positivity and $13 \%$ only anti-CCP positivity. At baseline, 64 (55.2\%) patients had an erosive disease and 50 (43.1\%) had extra-articular manifestations. Compared to the baseline (160 [74.8-496]), FR levels decreased significantly at 12 months (121 [49.1-321.8]) and 24 months (107.5 [43.3-332]) with $p=0.017$ and $p=0.029$, respectively. There were no differences in anti-CCP levels over time. No correlation was found between FR/anti-CCP levels and different DAS28 variables, DAS28 (4v) delta, HAQ, and HAQ delta at 12 months and 24 months.

\section{CONCLUSION}

We found that in patients with RA treated with a first anti-TNF- agent as first biologic, FR levels decreased at 12 months and 24 months follow-up. However, our study failed to demonstrate a correlation between autoantibodies levels and disease activity and HAQ variables. In fact, previous research demonstrated that there is an association between autoantibodies levels and disease activity in RA, nonetheless not being static and increasing with signs of inflammation at baseline. So, further research with large samples is needed to explore this correlation considering the adjustment for confounding inflammatory variables, namely number of swollen/tender joints and morning stiffness.

\section{KEYWORDS}

Rheumatoid arthritis, Autoantibodies, Antitumor necrosis factor alpha agent, Anticitrullinated protein antibodies, Rheumatoid factor. 